\title{
TWO-FLUX AND GREEN'S FUNCTION METHOD FOR TRANSIENT RADIATIVE TRANSFER IN A SEMITRANSPARENT LAYER
}

\author{
Robert Siegel \\ Lewis Research Academy \\ NASA Lewis Research Center, Cleveland, Ohio, USA
}

\begin{abstract}
A method using a Green's function is developed for computing transient temperatures in a semitransparent layer by using the two-flux method coupled with the transient energy equation. Each boundary of the layer is exposed to a hot or cold radiative environment, and is heated or cooled by convection. The layer refractive index is larger than one, and the effect of internal reflections is included with the boundaries assumed diffuse. The analysis accounts for internal emission, absorption, heat conduction, and isotropic scattering. Spectrally dependent radiative properties are included, and transient results are given to illustrate two-band spectral behavior with optically thin and thick bands. Transient results using the present Green's function method are verified for a gray layer by comparison with a finite difference solution of the exact radiative transfer equations; excellent agreement is obtained. The present method requires only moderate computing times and incorporates isotropic scattering without additional complexity. Typical temperature distributions are given to illustrate application of the method by examining the effect of strong radiative heating on one side of a layer with convective cooling on the other side, and the interaction of strong convective heating with radiative cooling from the layer interior.
\end{abstract}

\section{NOMENCLATURE}

a absorption coefficient of semitransparent medium, $\mathrm{m}^{-1}$

c specific heat of semitransparent medium, $\mathrm{J} / \mathrm{kg} \cdot \mathrm{K}$

$\mathrm{C}_{1} \ldots \mathrm{C}_{6}$ coefficients in Green's function

$D$ thickness of semitransparent layer, $m$

$\mathrm{E}_{1}, \mathrm{E}_{2}$ coefficients in homogeneous solution

$F_{L} \quad$ blackbody energy fraction at $t(X, \tau)$ in large frequency band

$F_{1 L}, F_{2 L}$ blackbody fractions of external radiation fluxes in large frequency band

g Green's function for radiative heat source

G the flux quantity $2\left(q_{r}^{+}+q_{r}^{-}\right), W / m^{2} ; \tilde{G}=G / \sigma T_{j}^{4}$

$h_{1}, h_{2}$ convective heat transfer coefficients at $x=0, D, W / m^{2} K$

$\mathrm{H}_{1}, \mathrm{H}_{2}$ dimensionless parameters, $h_{1} / \sigma T_{i}^{3}$ and $h_{2} / \sigma T_{i}^{3}$

k thermal conductivity of layer, $\mathrm{W} / \mathrm{m} \cdot \mathrm{K}$

m the quantity $\left[3 \mathrm{~K}_{\mathrm{D}}^{2}(1-\Omega)\right]^{1 / 2}$

n refractive index of radiating medium

$\mathrm{N}$ conduction-radiation parameter, $\mathrm{k} / 4 \sigma \mathrm{T}_{\mathrm{i}}^{3} \mathrm{D}$

$P \quad$ the quantity $\left(2 / 3 k_{\mathrm{p}}\right)\left[\left(1+\rho^{i}\right) /\left(1-\rho^{i}\right)\right]$

$\mathrm{q}_{\mathrm{r}} \quad$ radiative flux in the $\mathrm{x}$-direction, $\mathrm{W} / \mathrm{m}^{2} ; \tilde{\mathrm{q}}_{\mathrm{r}}=\mathrm{q}_{\mathrm{r}} / \sigma \mathrm{T}_{\mathrm{i}}^{4}$

$\mathrm{q}_{\mathrm{r}}^{+}, \mathrm{q}_{\mathrm{r}}^{-} \quad$ radiative fluxes in positive and negative $\mathrm{x}$ directions, $\mathrm{W} / \mathrm{m}^{2}$

$\mathrm{q}_{\mathrm{r} 1}, \mathrm{q}_{\mathrm{r} 2}$ external radiation fluxes $\sigma \mathrm{T}_{\mathrm{s1}}^{4}$ and $\sigma \mathrm{T}_{\mathrm{s2}}^{4}$ incident at $\mathrm{x}=0$ and $\mathrm{D}, \mathrm{W} / \mathrm{m}^{2}$

$\tilde{\mathrm{q}}_{\mathrm{r} 1}, \mathrm{q}_{\mathrm{r} 2}$ dimensionless radiation fluxes, $\mathrm{q}_{\mathrm{r} 1} / \sigma \mathrm{T}_{\mathrm{i}}^{4}=\left(\mathrm{T}_{\mathrm{s} 1} / \mathrm{T}_{\mathrm{i}}\right)^{4}$ and $\mathrm{q}_{\mathrm{r} 2} / \sigma \mathrm{T}_{\mathrm{i}}^{4}=\left(\mathrm{T}_{\mathrm{s} 2} / \mathrm{T}_{\mathrm{j}}\right)^{4}$ 
$\mathrm{T}$ absolute temperature, $\mathrm{K} ; \mathrm{t}=\mathrm{T} / \mathrm{T}_{\mathrm{i}}$

$T_{\mathfrak{k} 1}, T_{\mathfrak{g} 2}$ gas temperatures for convection at $\mathrm{x}=0$ and $\mathrm{D}, \mathrm{K}$

$t_{21}, t_{82}$ dimensionless gas temperatures, $T_{81} / T_{i}, T_{22} / T_{i}$

$T_{i} \quad$ initial uniform temperature (used as a reference temperature), $K$

$T_{s 1}, T_{22}$ temperatures of blackbody radiative surroundings at $x=0$ and $D, K$

$x \quad$ coordinate in direction across layer, $m ; X=x / D$

$\theta$ time, $s$

$\boldsymbol{K}_{\mathrm{D}} \quad$ optical thickness of layer, $\left(\mathrm{a}+\sigma_{\mathfrak{a}}\right) \mathrm{D}$

$\lambda_{c} \quad$ cutoff wavelength in vacuum, $\mu \mathrm{m}$

$v$ radiation frequency, $s^{-1}$

$\rho$ density of semitransparent medium, $\mathrm{kg} / \mathrm{m}^{3}$

$\rho^{\circ}, \rho^{i}$ external and internal reflectivities at a boundary

$\sigma \quad$ Stefan-Boltzmann constant, $\mathrm{W} / \mathrm{m}^{2} \mathrm{~K}^{4}$

$\sigma$ scattering coefficient in layer, $\mathrm{m}^{-1}$

$\tau$ dimensionless time, $\left(4 \sigma T_{i}^{3} / \rho c D\right) \theta$

$\Omega \quad$ scattering albedo, $\sigma_{d}\left(a+\sigma_{\mathrm{z}}\right)$

Subscripts

L,S spectral bands with large and small frequencies

\section{INTRODUCTION}

For semitransparent materials where thermal radiation affects internal temperature distributions, transient behavior has been studied much less than steady state. There is, however, a significant need for detailed transient solutions to examine heat transfer and thermal stress behavior of ceramic components during reentry, thermal protection coatings, tempering of glass windows, porous burners and insulation systems, liquid drop space radiators, and some high temperature components in advanced aircraft engines. For these applications, internal emission is large, and unknown temperature distributions must be determined that are time accurate. The references considered here are for solid materials that have an index of refraction larger than one so internal reflections occur within the semitransparent region.

An important early analysis was on the heat treatment of glass. Specular interface reflections were included, and two spectral absorption bands were used along with an opaque region for wavelengths larger than $4 \mu \mathrm{m}$. An analysis of transient temperature distributions in spacecraft windows for the thermal environment during reentry ${ }^{2}$ followed somewhat along the earlier principles! A zonal method was used with a quadratic temperature distribution assumed in each zone. Some experimental verification was obtained by exposing single-pane windows to a high temperature radiation source and measuring transient surface temperatures. For a similar application, a recent study is for a silica window in high speed flow. Recent work ${ }^{4}$ also provides additional experimental verification of transient temperature predictions for a glass plate. The plate was initially heated to a high temperature, and was then exposed to the ambient room on one side and to free convection and a radiant heater on the other side. Internal radiation effects were found to be very significant, as predicted by a two-band spectral calculation. Additional calculations for glass plates ${ }^{3}$ used a nodal analysis based on a zonal method. General external boundary conditions were used with both radiation and convection.

The analyses for glass plates have radiation combined with heat conduction, and scattering is negligible. In contrast a study with scattering that does not involve heat conduction is the cooling of a layer of liquid drops for dissipation of waste heat in the cold vacuum of outer space. Isotropic scattering was included within the droplet cloud. The resulting integral equation for the radiative source function was solved by iteration at each time step, and a forward finite-difference extrapolation was used in time.

Many of the recent transient radiation analyses use some type of finite-difference method for solving the transient energy equation. The instantaneous local radiative heat source term in the energy equation has 
been evaluated by direct numerical integration, by using the two-flux equations, by various expansion methods, ${ }^{90}$ and with the differential approximation." A semi-explicit finite-difference procedure with a "fixed grid was used for a plane layer with scattering included." Some effects of anisotropic scattering on transients have been examined. Transient solutions using the exact radiative transfer equations ${ }^{7}$ are used to verify the accuracy of the present Green's function method. These results are for a layer with refractive index larger than one with external convection and radiation at each boundary?

Most of the transient studies have been one-dimensional, usually for single plane layers. For a multilayer composite," a hybrid numerical method was used; the spatial variation in temperature was solved with finite differences, and a fourth-order Runge-Kutta method was used to extrapolate forward in time. For a two-dimensional rectangular region," a modified differential approximation was used for the radiative portion; a Crank-Nicolson finite-difference procedure was used for the transient energy equation. A fully implicit finite-difference solution ${ }^{15}$ used an alternating direction implicit method for transient solutions in a two-dimensional square region. Two-dimensional Gaussian integration was used to evaluate the local radiative source in terms of the instantaneous temperature distribution.

The present analysis develops a Green's function solution for the radiative source term using the two-flux equations. Various multi-flux methods have been discussed as a simplification for computing the radiative flux term in the energy equation. ${ }^{16}$ For the general boundary conditions of external convection and radiation on a layer with diffuse interfaces and unspecified surface temperatures, it was shown that the two-flux method can be used to predict accurate temperature distributions and heat fluxes for steady state.7 By use of a shooting method to evaluate the radiative flux equations, the two-flux method was used to obtain transient solutions in materials with large refractive indices typical of ceramics. The shooting method, however, was limited to layer optical thicknesses less than about 5 where sufficient coupling exists between values at the two boundaries. Using the Green's function method, transient results are obtained here for optical thicknesses up to 40 and are compared with the opaque limit. An advantage of the two-flux method is that isotropic scattering is included without any additional complication. Typical transient results with large scattering are given to illustrate that scattering effects can be readily examined. The analytical relations are written for two spectral bands, and the form of the two-band equations shows how they can be expanded to include more detailed spectral variations.

\section{ANALYSIS}

A plane layer as shown in Fig. 1 has thickness $D$ and radiative absorption and scattering properties that depend on frequency. The layer material is a semitransparent absorbing dielectric such as a ceramic, that is heat conducting and isotropically scattering with a refractive index $n>1$. The layer boundaries are assumed sufficiently rough that they can be assumed diffuse. ${ }^{18}$ For the results given here and for the nondimensionalization used, the layer is initially at uniform temperature $T_{i}$, but the theory applies for an arbitrary initial temperature distribution where $T_{i}$ would then be a characteristic reference temperature. To begin the transient, the layer is subjected to surroundings so each boundary can receive radiative energy and is in contact with a gas that provides convective heating or cooling. Transient temperature distributions are obtained in the layer until steady-state is reached corresponding to the external radiation and convection conditions.

\subsection{Energy Equation and Two-Flux Relations for a Gray Layer}

The analysis is developed first for a gray layer and is then extended for spectral calculations. The energy equation in dimensionless form for the transient temperatures in the layer is ${ }^{7}$, 


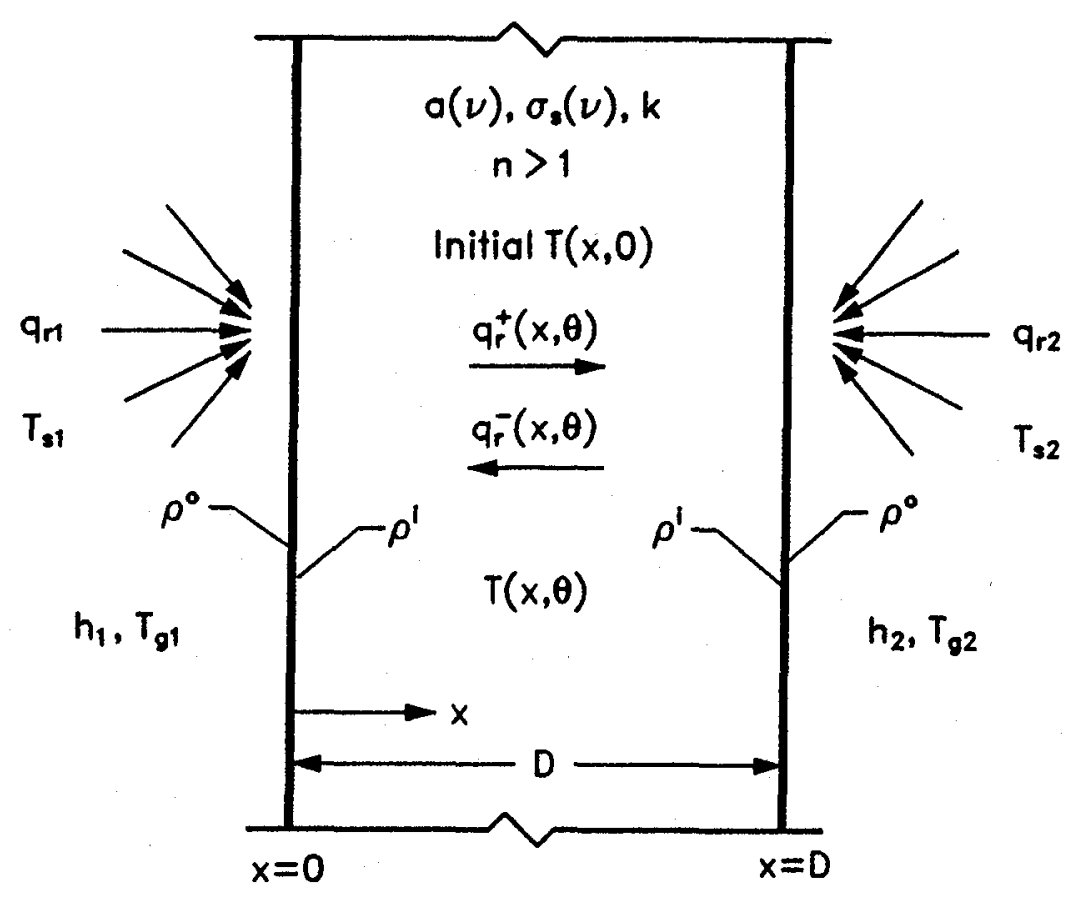

Figure 1. Geometry and nomenclature for transient radiation and conduction in a semitransparent layer with spectral properties and isotropic scattering.

$$
\frac{\partial t}{\partial \tau}=N \frac{\partial^{2} t}{\partial X^{2}}-\frac{1}{4} \frac{\partial \tilde{q}_{r}}{\partial X}
$$

where $-(1 / 4) \partial \tilde{\mathrm{q}}_{r}(\mathrm{X}, \tau) / \partial \mathrm{X}$ is the local radiative heat source arising from absorption, emission and scattering. Properties are assumed independent of temperature. The $\partial \widetilde{q}_{r}(X, \tau) / \partial X$ is obtained in terms of $t(X, \tau)$ from the two-flux relations which have been found to provide accurate results in previous steady-state ${ }^{17}$ and transient ${ }^{8}$ studies. The two-flux equations using the Milne-Eddington approximation are,

$$
\frac{\partial \tilde{q}_{r}(X, \tau)}{\partial X}=\kappa_{D}(1-\Omega)\left[4 n^{2} t^{4}(X, \tau)-\tilde{G}(X, \tau)\right]
$$

with $G(X, \tau)$ related to the radiative flux $\tilde{\mathrm{q}}_{r}(X, \tau)$ by,

$$
\frac{\partial \tilde{G}(X, \tau)}{\partial X}=-3 K_{D} \tilde{q}_{r}(X, \tau)
$$

The $\tilde{q}_{r}$ and $G$ are related to the positive and negative radiative fluxes shown in Fig. 1 by $\tilde{\mathrm{q}}_{\mathrm{r}}=\tilde{\mathrm{q}}_{\mathrm{r}}^{+}-\tilde{\mathrm{q}}_{\mathrm{r}}^{-}$and $G=2\left(\tilde{q}_{r}^{+}+\tilde{q}_{r}^{*}\right)$.

\subsection{Boundary and Initial Conditions}

For a semitransparent material there is radiant absorption only within the material interior so the conduction and convection conditions at the layer boundaries are

$$
\left.\frac{\partial t}{\partial X}\right|_{X=0}=-\frac{H_{1}}{4 N}\left[t_{g 1}-t(0, \tau)\right] \quad ;\left.\quad \frac{\partial t}{\partial X}\right|_{X=1}=-\frac{H_{2}}{4 N}\left[t(1, \tau)-t_{g 2}\right]
$$

The radiative boundary conditions must include the effects of external and internal reflections at the surfaces that are subjected to dimensionless external diffuse fluxes $\tilde{q}_{1}$ and $\tilde{q}_{12}$. By considering the incident 
and reflected fluxes at and across an interface, the following boundary relations between $\tilde{G}$ and $\tilde{q}_{r}$ were developed,

$$
\begin{aligned}
& \tilde{G}(0, \tau)=4 \frac{1-\rho^{0}}{1-\rho^{i}} \tilde{q}_{r 1}-2 \frac{1+\rho^{i}}{1-\rho^{i}} \tilde{q}_{r}(0, \tau) \\
& \tilde{G}(1, \tau)=4 \frac{1-\rho^{0}}{1-\rho^{i}} \tilde{q}_{r 2}+2 \frac{1+p^{i}}{1-\rho^{i}} \tilde{q}_{r}(1, \tau)
\end{aligned}
$$

To begin the transient solution, the specified initial condition for the results given here is a uniform temperature $T(x, 0)=T_{i}$ so $t(X, 0)=1$, but the method developed is valid for an arbitrary $t(X, 0)$. An initial distribution is also needed for $\partial \widetilde{q}_{r}(X, 0) / \partial X$. This is found by solving Eqs. (2a) and (2b) with $t(X, \tau)=t(X, 0)$, and the result is conveniently obtained from the Green's function solution that is developed. After substituting $\partial \tilde{\mathrm{q}}_{\mathrm{r}}(\mathrm{X}, 0) / \partial \mathrm{X}$ into the energy equation, the transient solution is advanced one time increment by an implicit finite-difference solution. The new temperature distribution, $t(X, \Delta \tau)$, is inserted into the Green's function solution to obtain $\partial \tilde{q}_{r}(X, \Delta \tau) / \partial X$ and the energy equation is then solved implicitly for $\mathrm{t}(\mathrm{X}, 2 \Delta \tau)$. The process is repeated until steady conditions are achieved. The sections that follow will develop the Green's function solution and the implicit finite-difference solution of the energy equation.

\subsection{Green's Function for $\partial \bar{q}_{r}(X, \tau) / \partial X$ for a Gray Layer}

The Green's function is developed for a gray layer and is then extended in the next section for spectral calculations. Equation (2b) is differentiated in $X$ and is used to eliminate $\partial \tilde{q}_{r}(X, \tau) / \partial X$ from Eq. (2a); this yields a second order equation for $\dot{G}(X, \tau)$,

$$
\frac{d^{2} \tilde{G}}{d X^{2}}-m^{2} \tilde{G}(X, \tau)=-4 m^{2} n^{2} t^{4}(X, \tau)
$$

where $m^{2}=3 k_{D}^{2}(1-\Omega)$. The $\tau$ functional notation is omitted for convenience in what follows. The homogeneous part of Eq. (5) is satisfied by $\cosh (\mathrm{mX})$ and $\sinh (\mathrm{mX})$ so the Green's function must have the form,

$$
g(X, \xi)= \begin{cases}C_{1}(\xi) \cosh m X+C_{2}(\xi) \sinh m X & 0 \leq X<\xi \\ C_{3}(\xi) \cosh m X+C_{4}(\xi) \sinh m X & \xi<X \leq 1\end{cases}
$$

To obtain the coefficients $\mathrm{C}_{1}(\xi) \ldots \mathrm{C}_{4}(\xi)$, the boundary conditions $(4 a, b)$ are written in terms of $\mathrm{G}$ by using Eq. (2b),

$$
\begin{aligned}
& \tilde{G}(0)=4 \frac{1-\rho^{0}}{1-\rho^{i}} \tilde{q}_{r 1}+\left.\frac{2}{3 k_{D}} \frac{1+\rho^{i}}{1-\rho^{i}} \frac{d \tilde{G}}{d X}\right|_{X=0} \\
& \tilde{G}(1)=4 \frac{1-\rho^{0}}{1-\rho^{i}} \tilde{q}_{r 2}-\left.\frac{2}{3 x_{D}} \frac{1+\rho^{i}}{1-\rho^{i}} \frac{d \tilde{G}}{d X}\right|_{X=1}
\end{aligned}
$$

The $g(X, \xi)$ in Eq. (6) must satisfy the homogeneous forms of Eqs. (7a,b),

$$
g(0)-\left.\frac{2}{3 k_{D}} \frac{1+\rho^{i}}{1-\rho^{i}} \frac{d g}{d X}\right|_{X=0}=0 \quad ; \quad g(1)+\left.\frac{2}{3 k_{D}} \frac{1+\rho^{i}}{1-\rho^{i}} \frac{d g}{d X}\right|_{X=1}=0
$$


By using Eq. (6) in Eqs. (8a,b) to evaluate $C_{1}(\xi) \ldots C_{4}(\xi)$, the $g(X, \xi)$ is obtained in the form

$$
g(X, \xi)= \begin{cases}C_{5}(\xi)[\sinh m X+P m \cosh m X] & 0 \leq X<\xi \\ C_{6}(\xi)[\sinh m(1-X)+P m \cosh m(1-X)] & \xi<X \leq 1\end{cases}
$$

where $P=\left(2 / 3 \kappa_{D}\right)\left[\left(1+\rho^{i}\right) /\left(1-\rho^{i}\right)\right]$.

The $C_{5}(\xi)$ and $C_{6}(\xi)$ are obtained by applying the two conditions ${ }^{19}$ that $g(X, \xi)$ is continuous at $X=\xi$, and that with increasing $X$ the derivative $\partial g(X, \xi) / \partial X$ passes through a discontinuity of -1 at $X=\xi$. This yields the two relations,

$C_{6}(\xi)[\sinh m(1-\xi)+P m \cosh m(1-\xi)]=C_{5}(\xi)[\sinh m \xi+P m \cosh m \xi]$

$C_{6}(\xi)\left[-m \cosh m(1-\xi)-P_{m}^{2} \sinh m(1-\xi)\right]$

$-C_{5}(\xi)\left[m \cosh m \xi+P m^{2} \sinh m \xi\right]=-1$

Equations $(10 a, b)$ are solved for $C_{5}(\xi)$ and $C_{6}(\xi)$, and after substituting into Eq. (9) this gives the final form of the Green's function as,

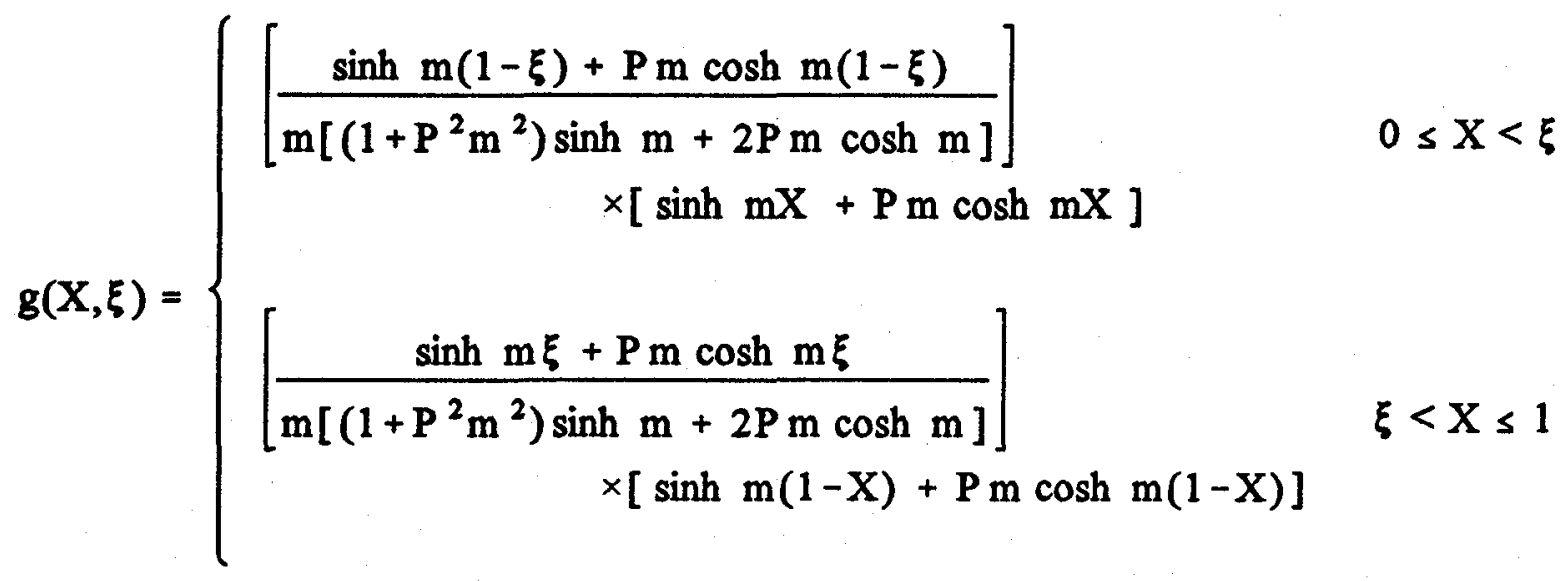

The $g(X, \xi)$ in Eq. (11) will be used to account for the nonhomogeneous term in Eq. (5) when computing $G(X, \tau)$ at each time step during the transient calculations. To obtain the complete solution for $G(X, \tau)$ the solution for the homogeneous part of Eq. (5) is needed. This is of the form $G_{h}(X)=E_{1} \sinh (m X)+$ $E_{2} \cosh (m X)$. The boundary conditions Eq. $(7 a, b)$ are applied to evaluate $E_{1}$ and $E_{2}$ with the result

$$
\begin{aligned}
\tilde{G}_{h}(X)= & 4 \frac{1-\rho^{0}}{1-\rho^{i}} \frac{1}{\left(1+P^{2} m^{2}\right) \sinh m+2 P m \cosh m}\{[\sinh m(1-X) \\
& \left.+P m \cosh m(1-X)] \tilde{q}_{r 1}+[\sinh m X+P m \cosh m X] \tilde{q}_{r 2}\right\}
\end{aligned}
$$

Then adding $G_{h}(X)$ and the nonhomogeneous solution obtained with $g(X, \xi)$, the general solution of Eq. (5) at each time step is

$$
\tilde{G}(X, \tau)=\tilde{G}_{h}(X)+4 m^{2} n^{2} \int_{0}^{1} g(X, \xi) t^{4}(\xi, \tau) d \xi
$$

The $\tilde{G}(X, \tau)$ is substituted into Eq. (2a) along with $t^{4}(X, \tau)$ to evaluate $\partial \tilde{q}_{r}(X, \tau) / \partial X$ which is used in Eq. (1) to solve for the temperature distribution at the next time step. 


\subsection{Relations for a Two-Band Spectral Calculation}

- The relations developed for a gray material can be further developed for transient spectral solutions. To show how this is done, relations for two spectral bands are given. They are readily extended to more bands. Letting $L$ and $S$ designate bands with large and small frequencies, the radiative term needed for Eq. (1) is the sum of the large and small frequency contributions,

$$
\frac{\partial \tilde{q}_{r}(X, \tau)}{\partial X}=\frac{\partial \tilde{q}_{r L}(X, \tau)}{\partial X}+\frac{\partial \tilde{q}_{r S}(X, \tau)}{\partial X}
$$

From Eq. (2a),

$$
\frac{\partial \tilde{q}_{r L}(X, \tau)}{\partial X}=\kappa_{D L}\left(1-\Omega_{L}\right)\left[4 n^{2} t^{4}(X, \tau) F_{L}(t)-\tilde{G}_{L}(X, \tau)\right]
$$

where $F_{L}(t)$ is the blackbody fraction at $t(X, \tau)$ in the large frequency band. A similar relation is written for $\partial \tilde{q}_{\mathrm{s}} / \partial X$ for the small frequency band. The $\mathrm{G}_{\mathrm{L}}(\mathrm{X}, \tau)$ for Eq. (15) is found from Eq. (13) written for the $\mathrm{L}$ band,

$$
\tilde{G}_{L}(X, \tau)=\tilde{G}_{h L}(X)+4 m_{L}^{2} n^{2} \int_{0}^{1} g_{L}(X, \xi) t^{4}(\xi, \tau) F_{L}(t) d \xi
$$

and similarly for $\mathrm{G}_{\mathrm{s}}(\mathrm{X}, \tau)$. The homogeneous solution needed for Eq. (16) for the $\mathrm{L}$ band is obtained from Eq. (12) as

$$
\begin{aligned}
\tilde{G}_{b L}=4 \frac{1-\rho^{0}}{1-\rho^{i}} & {\left[\frac{1}{\left(1+P_{L}^{2} m_{L}^{2}\right) \sinh m_{L}+2 P_{L} m_{L} \cosh m_{L}}\right] } \\
\times & \left\{\left[\sinh m_{L}(1-X)+P_{L} m_{L} \cosh m_{L}(1-X)\right] \tilde{q}_{r 1} F_{1 L}\right. \\
+ & {\left.\left[\sinh m_{L} X+P_{L} m_{L} \cosh m_{L} X\right] \tilde{q}_{r 2} F_{2 L}\right\} }
\end{aligned}
$$

The Green's function in Eq. (16) for the L band is obtained from Eq. (11) as,

$$
g_{L}(X, \xi)=\left\{\begin{array}{cc}
{\left[\begin{array}{c}
\sinh m_{L}(1-\xi)+P_{L} m_{L} \cosh m_{L}(1-\xi) \\
m_{L}\left[\left(1+P_{L}^{2} m_{L}^{2}\right) \sinh m_{L}+2 P_{L} m_{L} \cosh m_{L}\right]
\end{array}\right]} & 0 \leq X<\xi \\
\times\left[\sinh m_{L} X+P_{L} m_{L} \cosh m_{L} X\right] & \\
{\left[\begin{array}{c}
\sinh m_{L} \xi+P_{L} m_{L} \cosh m_{L} \xi \\
m_{L}\left[\left(1+P_{L}^{2} m_{L}^{2}\right) \sinh m_{L}+2 P_{L} m_{L} \cosh m_{L}\right]
\end{array}\right.} & \xi<X \leq 1 \\
\times\left[\sinh m_{L}(1-X)+P_{L} m_{L} \cosh m_{L}(1-X)\right] &
\end{array}\right.
$$

Similar relations are written for the $S$ band. With these relations Eq. (15) can be evaluated for the $L$ band, and similarly for the S band; then $\partial \tilde{q}_{r} / \partial X$ is obtained from Eq. (14) for use in Eq. (1). 


\subsection{Numerical Solution}

"Starting with the initial $t(X, 0)$, the Green's function is used to obtain $G(X, 0)$, and the $\partial \tilde{q}_{r}(X, 0) / \partial X$ is evaluated from Eq. (2a) or Eq. (14) as described. Using Eq. (1), $t(X, \tau)$ is integrated forward in time using the following implicit finite-difference algorithm at the interior points of a uniform grid:

$$
\begin{aligned}
-\frac{N \Delta \tau}{(\Delta X)^{2}} t(X-\Delta X, \tau+\Delta \tau)+\left[1+\frac{2 N \Delta \tau}{(\Delta X)^{2}}\right] t(X, \tau+\Delta \tau) & -\frac{N \Delta \tau}{(\Delta X)^{2}} t(X+\Delta X, \tau+\Delta \tau) \\
& =t(X, \tau)-\left.\frac{\Delta \tau}{4} \frac{\partial \tilde{q}_{r}}{\partial X}\right|_{X, \tau}
\end{aligned}
$$

At the boundaries Eq. (19) is applied and the $t(-\Delta X, \tau+\Delta \tau)$ and $t(1+\Delta X, \tau+\Delta \tau)$ that appear are eliminated by using the boundary conditions Eqs. $(3 a, b)$ in the forms,

$$
\begin{aligned}
& \frac{t(\Delta X, \tau+\Delta \tau)-t(-\Delta X, \tau+\Delta \tau)}{2 \Delta X}=-\frac{H_{1}}{4 N}\left[t_{g 1}-t(0, \tau+\Delta \tau)\right] \\
& \frac{t(1+\Delta X, \tau+\Delta \tau)-t(1-\Delta X, \tau+\Delta \tau)}{2 \Delta X}=-\frac{H_{2}}{4 N}\left[t(1, \tau+\Delta \tau)-t_{g 2}\right]
\end{aligned}
$$

This yields the special relations at $\mathrm{X}=0$ and 1 as,

$$
\begin{array}{r}
{\left[1+\frac{2 \mathrm{~N} \Delta \tau}{(\Delta \mathrm{X})^{2}}+\frac{\mathrm{H}_{1} \Delta \tau}{2 \Delta \mathrm{X}}\right] \mathrm{t}(0, \tau+\Delta \tau)-\frac{2 \mathrm{~N} \Delta \tau}{(\Delta \mathrm{X})^{2}} \mathrm{t}(\Delta \mathrm{X}, \tau+\Delta \tau)} \\
=\mathrm{t}(0, \tau)-\left.\frac{\Delta \tau}{4} \frac{\partial \tilde{\mathrm{q}}_{\tau}}{\partial \mathrm{X}}\right|_{0, \tau}+\frac{\mathrm{H}_{1} \Delta \tau}{2 \Delta \mathrm{X}} \mathrm{t}_{\mathrm{g} 1} \\
-\frac{2 \mathrm{~N} \Delta \tau}{(\Delta \mathrm{X})^{2}} \mathrm{t}(1-\Delta \mathrm{X}, \tau+\Delta \tau)+\left[1+\frac{2 \mathrm{~N} \Delta \tau}{(\Delta \mathrm{X})^{2}}+\frac{\mathrm{H}_{2} \Delta \tau}{2 \Delta \mathrm{X}}\right] \mathrm{t}(1, \tau+\Delta \tau) \\
=\mathrm{t}(1, \tau)-\left.\frac{\Delta \tau}{4} \frac{\partial \tilde{\mathrm{q}}_{\tau}}{\partial X}\right|_{1, \tau}+\frac{\mathrm{H}_{2} \Delta \tau}{2 \Delta \mathrm{X}} \mathrm{t}_{\mathrm{g} 2}
\end{array}
$$

After advancing $t(X, \tau)$ each $\Delta \tau$ the radiant flux gradient is advanced to $\tau+\Delta \tau$ by using the Green's function solution in Eq. (2a) or (14). The $t(X, \tau)$ is then advanced to the next $\Delta \tau$ using Eqs. (19) and (21). After checking various grid sizes it was found that 81 evenly spaced points across the layer gave accurate solutions for $k_{D}<5$. For $k_{D}>5,161$ points were used which gave accurate solutions for $k_{D} \leq$ 40 A time increment of $\Delta \tau=0.0025$ provided stable and time accurate solutions for all results calculated here.

\section{RESULTS AND DISCUSSION}

The objective of this paper is to develop a convenient and accurate method for obtaining transient temperature distributions in a layer with isotropic scattering and spectrally dependent absorption and scattering properties. A Green's function solution was developed for the two-flux equations and this was used to obtain the radiative energy source term in the transient energy equation including externally 
incident radiation. The energy equation was solved with an implicit finite-difference method that included convective boundary conditions. The transient results that are presented serve two purposes. First the transient temperature distributions are compared with previous transient calculations using the exact equations of radiative transfer. This will verify the accuracy of the present transient two-flux Green's function method. The second purpose, as shown by the remaining figures, is to demonstrate some of the types of transient results that can be readily obtained with the present analytical and computational procedure.

The transient temperature distributions given here begin with a uniform initial temperature $t(X, 0)=1$, although the solution applies for any $t(X, 0)$. Figure 2 shows typical comparisons of transient two-flux results for $t(X, \tau)$ with those from a fully implicit method ${ }^{7}$ using the exact transfer equations to evaluate the radiative flux gradient in the transient energy equation. The layer is heated at the hot side $(X=0)$ by a radiative flux equal to that from blackbody surroundings at $T_{31}=1.5 \mathrm{~T}_{\mathrm{i}}$. At the other side $(X=1)$ the surroundings are at a low temperature $T_{\mathbf{2} 2}=0.5 \mathrm{~T}_{\mathrm{i}}$ so there is cooling by radiation through that boundary. The results in Fig. 2 illustrate the thermal behavior when there is convective cooling only on the side away from where radiative heating is applied. This simulates a possible condition for the wall of a combustion chamber when there is primarily radiative heating from combustion gases and soot on one side, and that side is not being film cooled.

The three parts of Fig. 2 are for optical thicknesses $x_{D}=2,10$ and 40 with no scattering and a refractive index, $n=2$. The temperature distributions from the present analysis are solid lines; the dashed lines in Figs. 2a,b were calculated from the exact transfer equations? When $\tau=2$ the $t(X, \tau)$ are within $1 \%$ of steady state. The convective cooling at $\mathrm{X}=1$ produces a temperature decrease near that boundary after the transient begins. The results from the present method agree very well with those from the exact transfer equations; excellent agreement throughout the transient is obtained for $k_{D}=10$.

The Green's function method can be applied for larger $\kappa_{D}$ values than were used for the exact solution? Computational difficulties often arise for large $\kappa_{D}$ because of the rapid local attenuation it provides. Transient temperatures for $k_{D}=40$ are in Fig. 2c. They are compared with $t(X, \tau)$ for an opaque layer where radiation is absorbed and emitted only at the boundaries, and internally there is only heat conduction. These limiting results were obtained by removing the internal radiation terms from the computer program and adding radiative absorption and emission to Eqs. $(3 a, b)$ for opaque boundaries. For $\tau=2$ the $t(X, \tau)$ in the opaque layer is within $1 \%$ of steady state; the distribution is slightly curved and it becomes a straight line as $\tau$ is further increased. The results show that for $k_{D}=40$ the layer is not thick enough to act as an opaque layer; there are very significant internal radiation effects on the transient and steady-state temperatures. A $K_{D}$ of at least 200 is required to approach the opaque limit.

In Fig. 3 comparisons are made with the exact transfer equations for a very different set of boundary conditions. The conditions are the same on both sides of the layer so the $t(X, \tau)$ are symmetric and only one-half of the distributions are shown. At the onset of the transient the gas temperatures are raised to two times the initial layer temperature, and there is strong convective heating at the boundaries with convection parameters $\mathrm{H}_{1}=\mathrm{H}_{2}=5$. The surrounding radiative temperatures are low and, as the layer temperature increases, this produces radiative cooling from the layer interior. This causes the interior temperatures to remain much lower than the surface temperature even as steady state is approached. These results are for $k_{\mathrm{D}}=1$ and they verify that the present method provides accurate solutions for a region of moderate optical thickness with large convective heating and interior radiative cooling. As shown in previous work ${ }^{7}$ where results are given for $n=1$, there are very significant effects of internal reflections when $n=2$ as used here.

An example of a transient two-band spectral calculation is in Fig. 4. At $\tau=0$ a radiative flux of $\tilde{q}_{r 1}=3^{4}$ is applied at $X=0$. The environment at $X=1$ and the convecting gases at both boundaries remain at the initial temperature, $T_{22}=T_{g 1}=T_{g 2}=T_{1}$. Hence, the layer is heated by radiation on one side with radiative cooling on the other side, and convective cooling at both sides. The steady state temperature distribution 


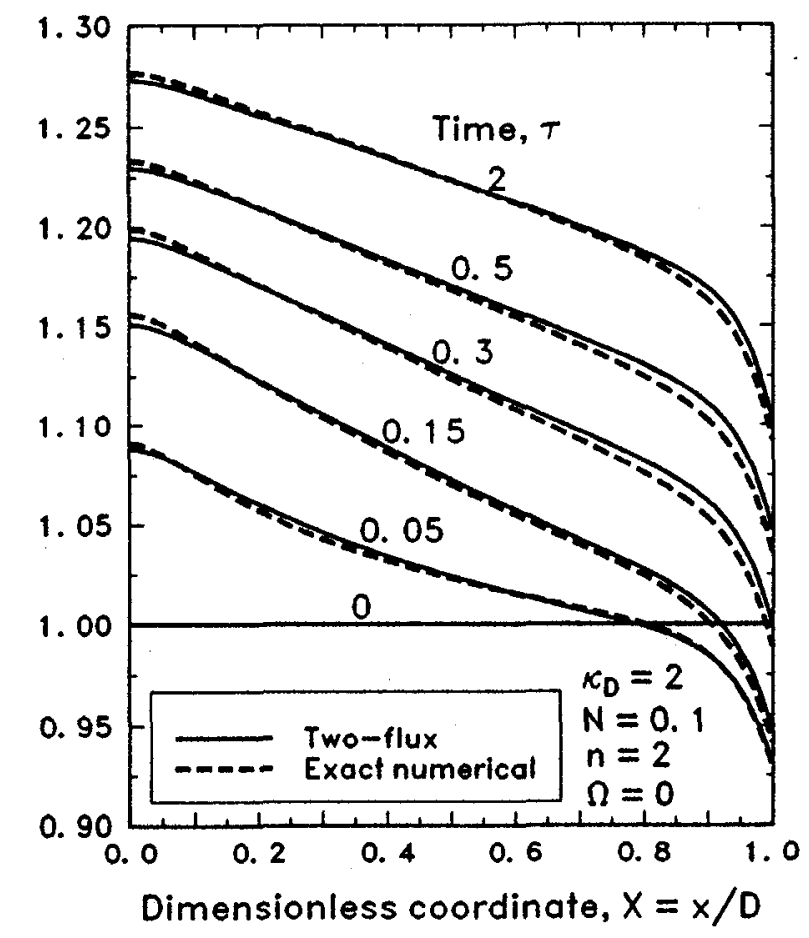

(a) Comparison with exact results for $K_{D}=2$.

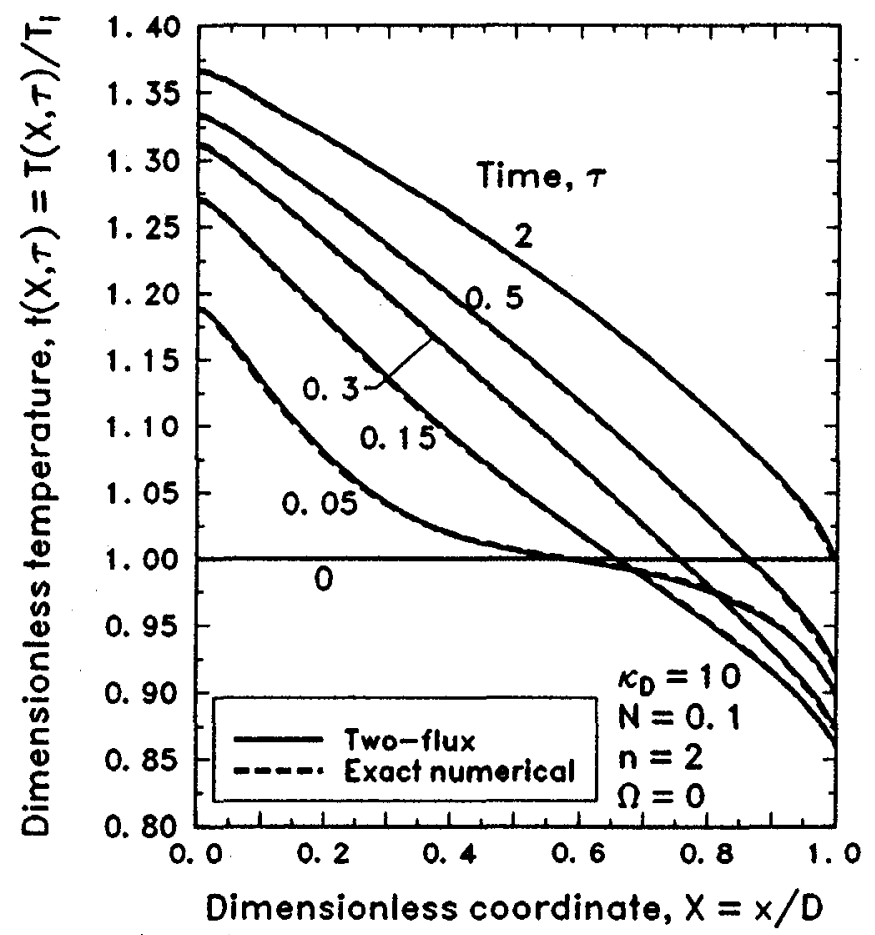

(b) Comparison with exact results for $K_{D}=10$.

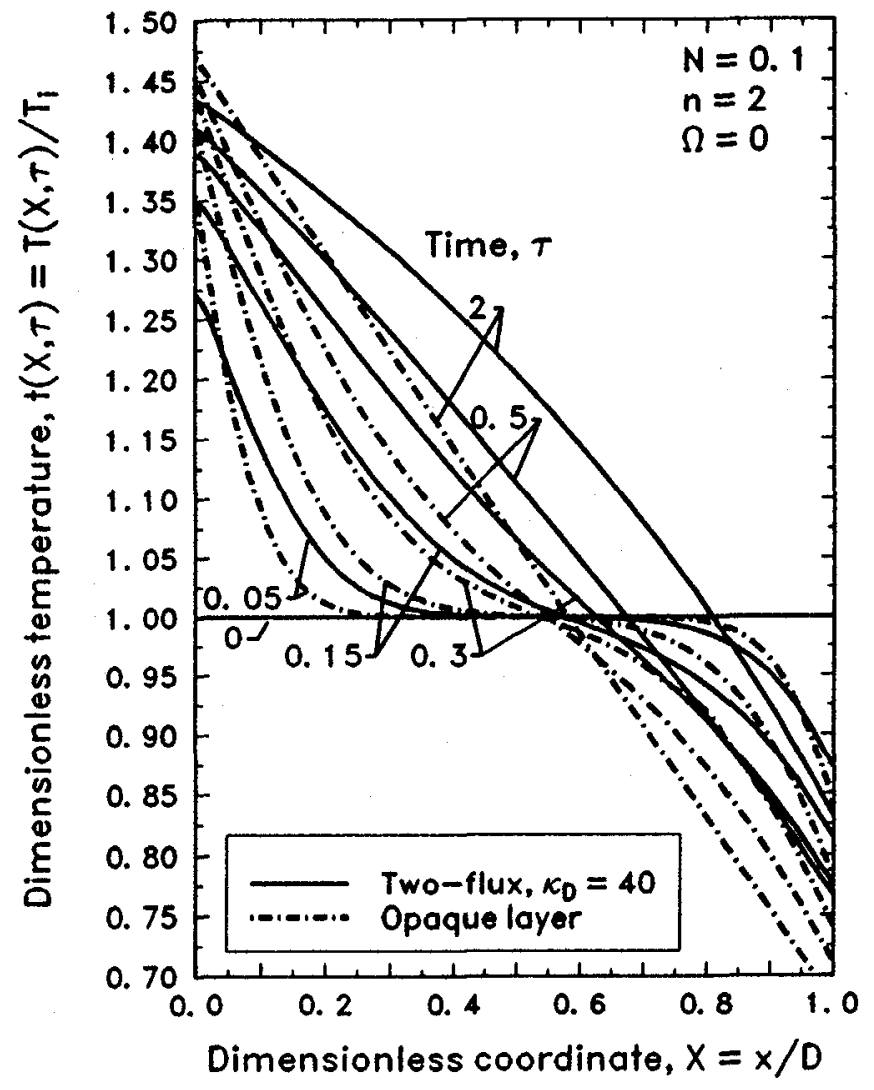

(c) Comparison of $K_{D}=40$ with opaque layer.

Figure 2. Two-flux, exact numerical, and opaque results for transient temperatures in a layer initially at uniform temperature after exposure to radiation on one side and convective cooling on the other.

$$
\text { Parameters: } \Omega=0, \mathrm{~N}=0.1, \mathrm{n}=2, \tilde{\mathrm{q}}_{\mathrm{r} 1}=1.5^{4}, \tilde{\mathrm{q}}_{\mathrm{r} 2}=0.5^{4}, \mathrm{H}_{1}=0, \mathrm{H}_{2}=1, \mathrm{t}_{\mathrm{g} 2}=0.5 \text {. }
$$

for an opaque layer is the long-dashed line, and compared with this line the final profiles reached in the transient analysis show there is a strong effect of internal radiation. The short-dash and dot-dash lines are for gray layers with $\kappa_{D}=1$ and 40 . The solid lines are for two bands, $\kappa_{D L}=1$ and $\kappa_{D S}=40$ with $\lambda_{c} T_{s 1}$ 


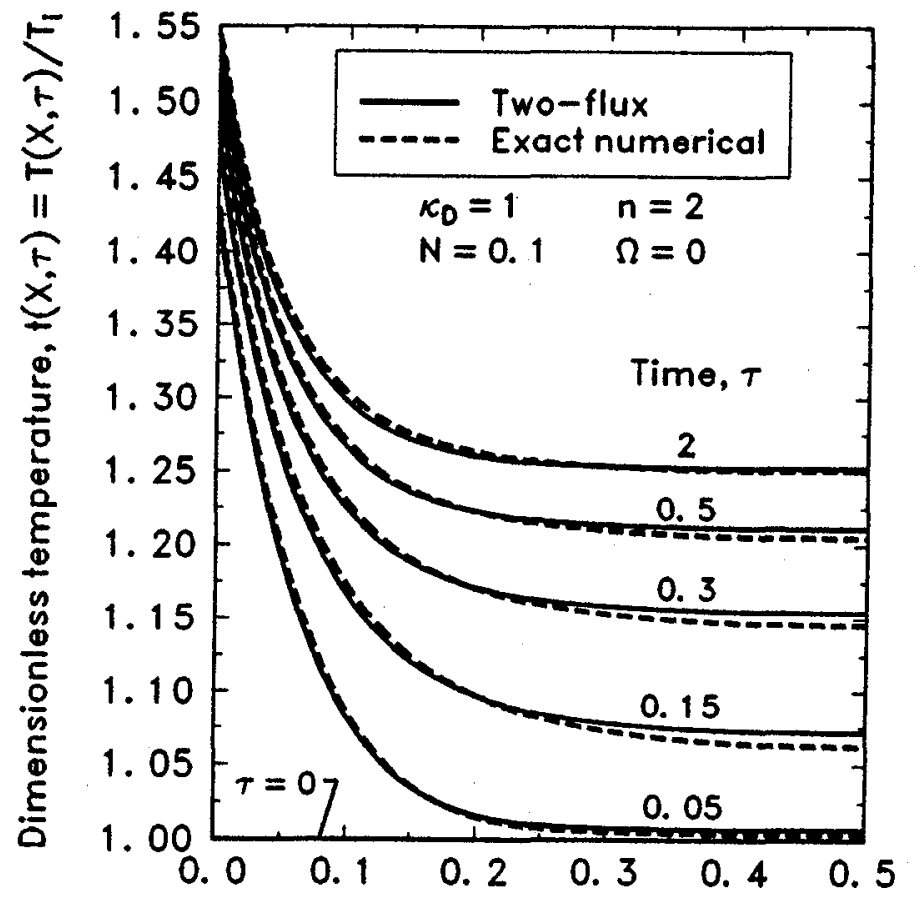

Dimensionless coordinate, $X=x / 0$

Figure 3. Two-flux and exact numerical results for transient temperatures in a layer initially at uniform temperature after exposure to hot gas convection on both sides with cold surroundings that produce internal radiative cooling. Parameters: $\Omega=0, \mathrm{k}_{\mathrm{D}}=1, \mathrm{~N}=0.1, \mathrm{n}=2, \tilde{\mathrm{q}}_{\mathrm{rl}}=$ $\mathrm{q}_{\mathrm{r} 2}=0.2^{4}, \mathrm{H}_{1}=\mathrm{H}_{2}=5, \mathrm{t}_{\mathrm{gl}}=\mathrm{t}_{\mathrm{g} 2}=2$.

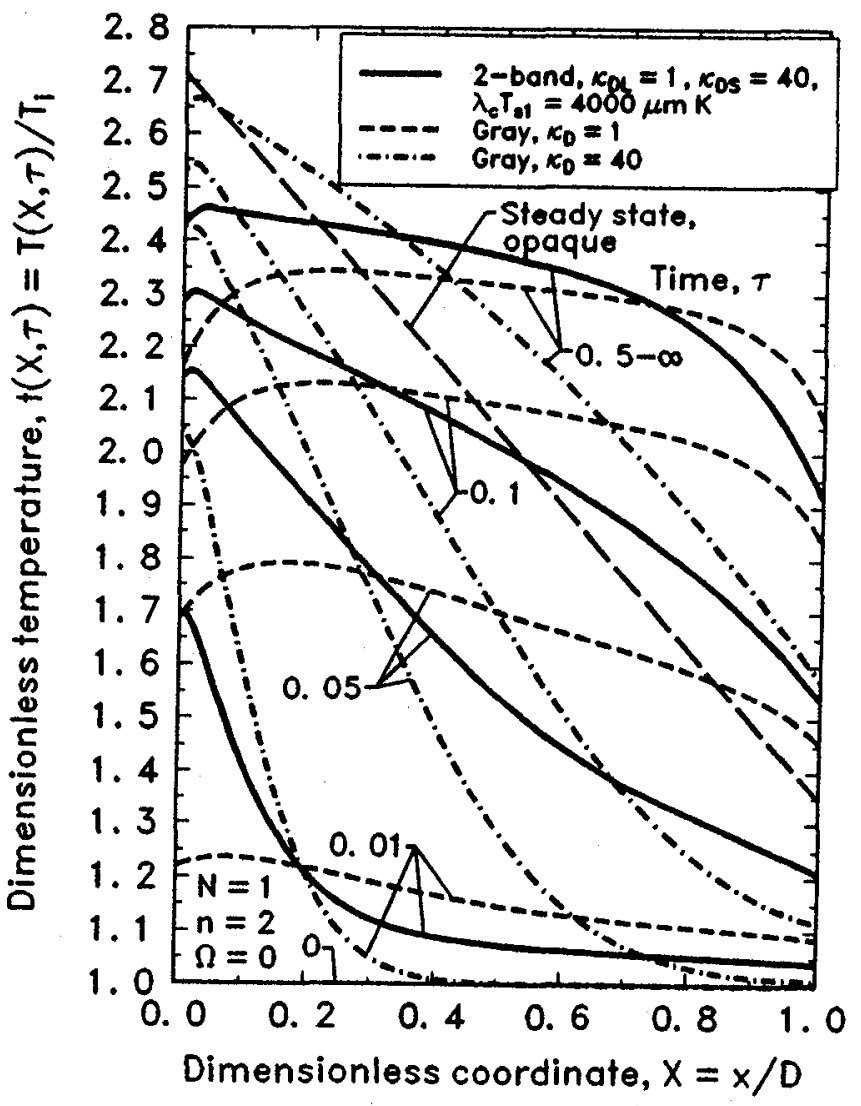

Figure 4. Two-flux transient temperatures in a layer initially at uniform temperature after exposure to radiative heating on one side and convective cooling at both sides with $\mathrm{T}_{\mathrm{g} 1}=\mathrm{T}_{\mathrm{g} 2}=$ $T_{i}$; results for two bands compared with gray results for each band optical thickness. Parameters: $\Omega=0, N=0.1, \mathrm{n}=2, \tilde{\mathrm{q}}_{\mathrm{r} 1}=3^{4}, \tilde{\mathrm{q}}_{\mathrm{r} 2}=$

$$
1, \mathrm{H}_{1}=\mathrm{H}_{2}=10, \mathrm{t}_{\mathrm{g} 1}=\mathrm{t}_{\mathrm{g} 2}=1 \text {. }
$$

$=4000 \mu \mathrm{m} \mathrm{K}$; this band cutoff places about one-half of the incident radiation at $\mathrm{T}_{31}$ in each band. The general behavior of the two-band results is to be between the gray distributions at each time except for some deviation from this behavior at steady state. This demonstrates the importance of including spectral property variations. With the present method this adds very little complexity, and computer time increased about $50 \%$.

The two-band results are continued in Fig. 5 where scattering is introduced with $\Omega=0.9$ or 0.99 in both bands. By raising $\Omega$ and keeping the same $\kappa_{\mathrm{D}}$, internal absorption is decreased and the transient temperatures do not respond as rapidly to the imposed radiative flux as for $\Omega=0$. This also yields decreased temperatures at steady state. Since scattering is included in the two-flux relations, it does not add to the computer time or difficulty in evaluating the solution developed here. This is in contrast with needing to solve the scattering source function integral equation when using the exact transfer relations.

In Fig. 6 the boundary conditions are of the type discussed for Fig. 3 where comparisons were made with results from the exact transfer equations. For all parts of Fig. 6 the layer is subjected for $\tau>0$ to a high temperature gas at $T_{g 1}=T_{g 2}=4 T_{i}$ so there is large convective heating on both sides. The surroundings are kept at $T_{31}=T_{12}=T_{i}$ so radiative cooling occurs from the layer interior as the temperatures rise. Since the imposed conditions are symmetric, the $t(X, \tau)$ are shown for one-half of the layer. The purpose of these figures is to illustrate that for a much different type of transient, spectral calculations including scattering can be readily carried out with the Green's function solution. The results in Fig. $6 \mathrm{a}$ are base 


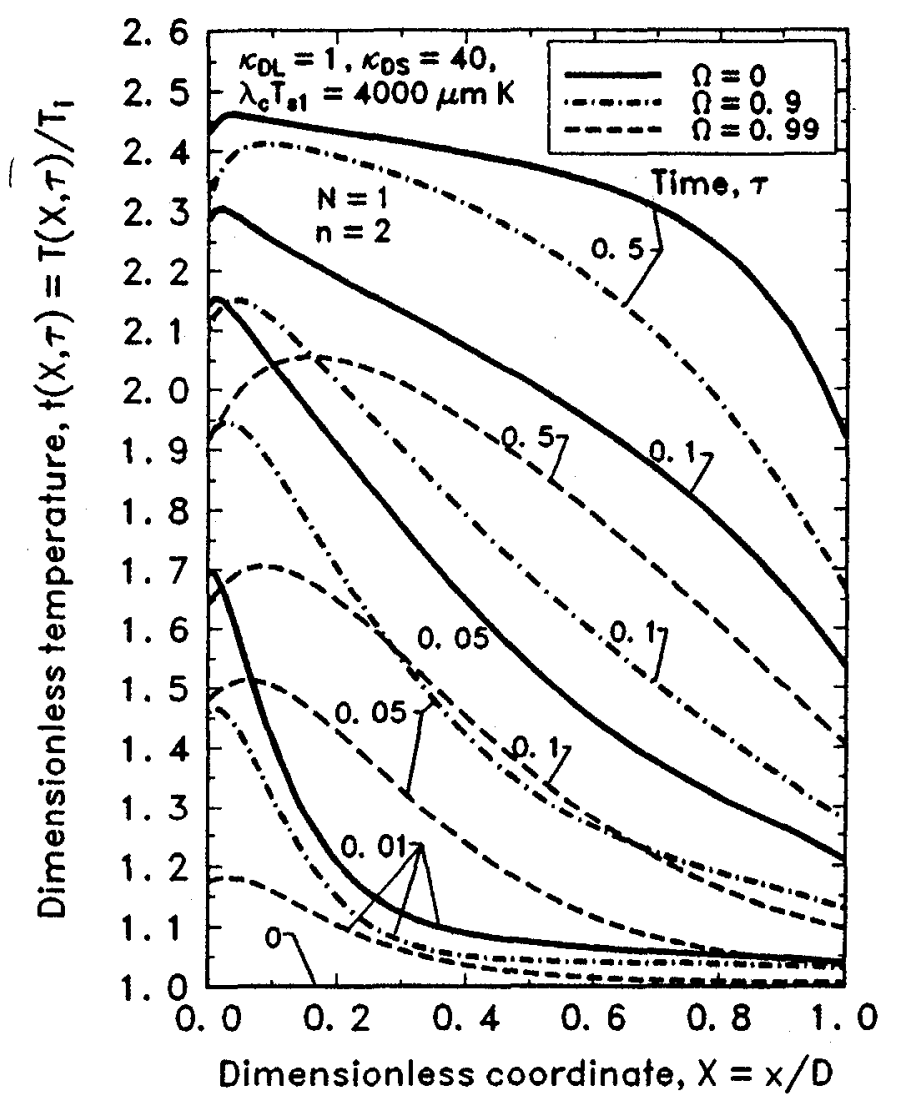

Figure 5. Two-flux transient temperatures in a layer initially at uniform temperature after exposure to radiative heating on one side and convective cooling at on both sides with $T_{g 1}=T_{g 2}=T_{i}$; results for two bands with large scattering compared with results for $\Omega=0$. Parameters: $N=1, \mathrm{n}=2, \tilde{\mathrm{q}}_{\mathrm{r} 1}=3^{4}, \tilde{\mathrm{q}}_{\mathrm{r} 2}=1$,

$$
\mathrm{H}_{1}=\mathrm{H}_{2}=10, \mathrm{t}_{\mathrm{g} 1}=\mathrm{t}_{\mathrm{g} 2}=1, \lambda_{\mathrm{c}} \mathrm{T}_{\mathrm{s1}}=4000 \mu \mathrm{m} \mathrm{K} \text {. }
$$

cases for gray layers with $\kappa_{\mathrm{D}}=0.1$ and 10. The uniform steady-state temperature is also shown (dot-dash line) for an opaque layer $\left(k_{D}-\infty\right)$. For $k_{D}=10$ the temperatures at $\tau=1$ (which are within less than $1 \%$ of steady state) are somewhat below the opaque limit except near the boundary; there is a small amount of radiative cooling from within the layer that provides lower interior temperatures. Radiative cooling has a very strong effect for $k_{\mathrm{D}}=0.1$ producing interior temperatures considerably below the surface temperature and the opaque limit.

Retaining the same parameters, Fig. $6 \mathrm{~b}$ demonstrates a two-band spectral calculation with $\boldsymbol{K}_{\mathrm{DL}}=0.1, \boldsymbol{k}_{\mathrm{DS}}$ $=10$, and $\lambda_{c} T_{s 1}=4000$ or $2000 \mu \mathrm{m} \mathrm{K}$. Decreasing $\lambda_{c} T_{s 1}$ places more energy in the $\kappa_{D s}$ band, and the transient results change their behavior somewhat like increasing $\kappa_{\mathrm{D}}$ for the gray results in Fig. $6 \mathrm{a}$. In Fig. $6 \mathrm{c}$ scattering is included for $\Omega=0.9$ and the parameters in Fig. $6 \mathrm{~b}$ with $\lambda_{\mathrm{c}} \mathrm{T}_{\mathrm{s}}=2000 \mu \mathrm{m} \mathrm{K}$. Since increasing $\Omega$ lowers the amount of absorption in the layer, there is an increased radiative cooling effect as was obtained for $K_{D}=0.1$ in Fig. $6 a$.

\section{CONCLUSIONS}

A method was developed using a Green's function solution in conjunction with the two-flux equations to obtain transient temperatures in a semitransparent layer including internal radiation absorption, emission, and scattering with heat conduction. Starting from an initial temperature distribution that can have any arbitrary shape, the layer is heated or cooled by external radiation and convection. Transient temperature distributions from the present method were compared with results where the radiative flux gradient in the transient energy equation was evaluated numerically from the exact radiative transfer equations and a fully implicit finite difference solution was used. Good agreement was obtained for 


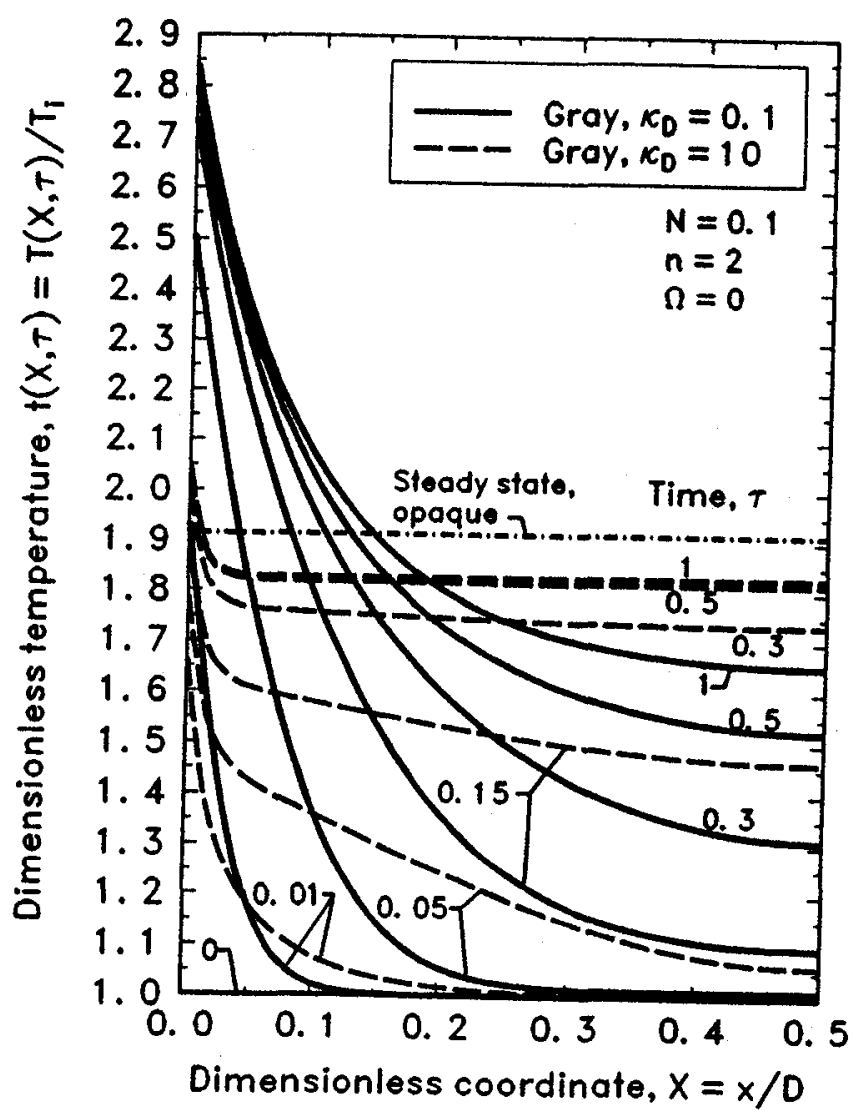

(a) Gray layers with $\kappa_{D}=0.1$ and 10 .

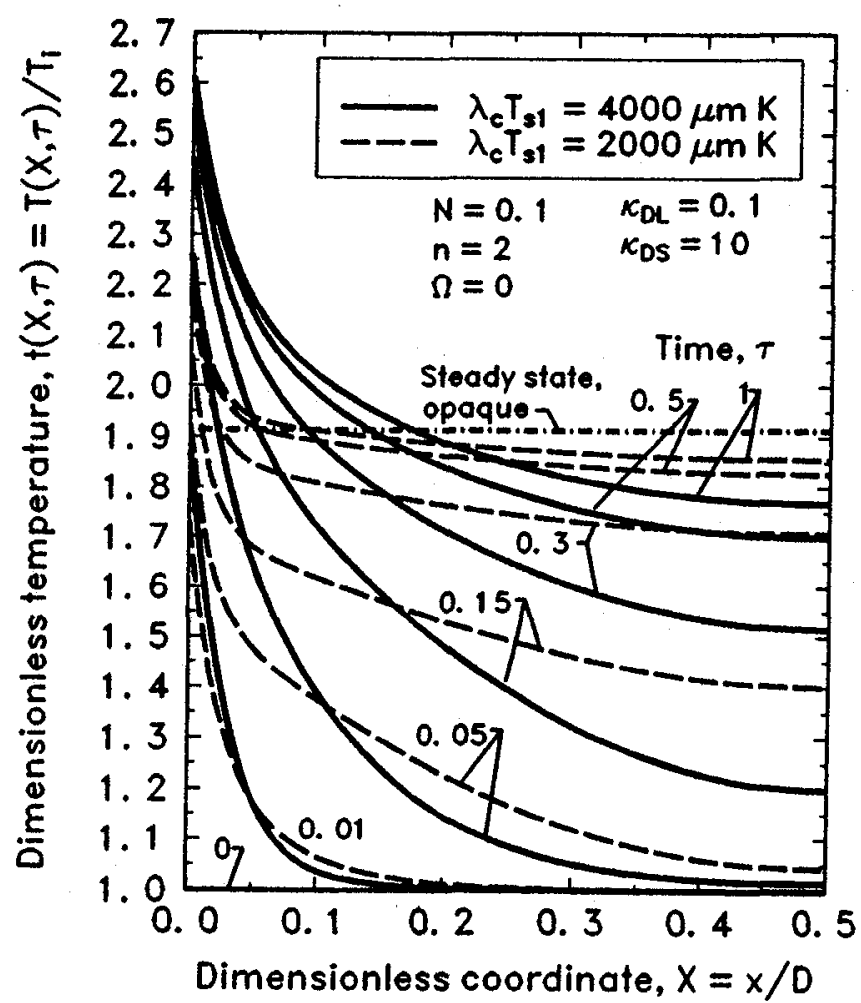

(b) Two bands with $\kappa_{D L}=0.1$ and $\kappa_{D S}=10$; comparison for two different band cutoffs.

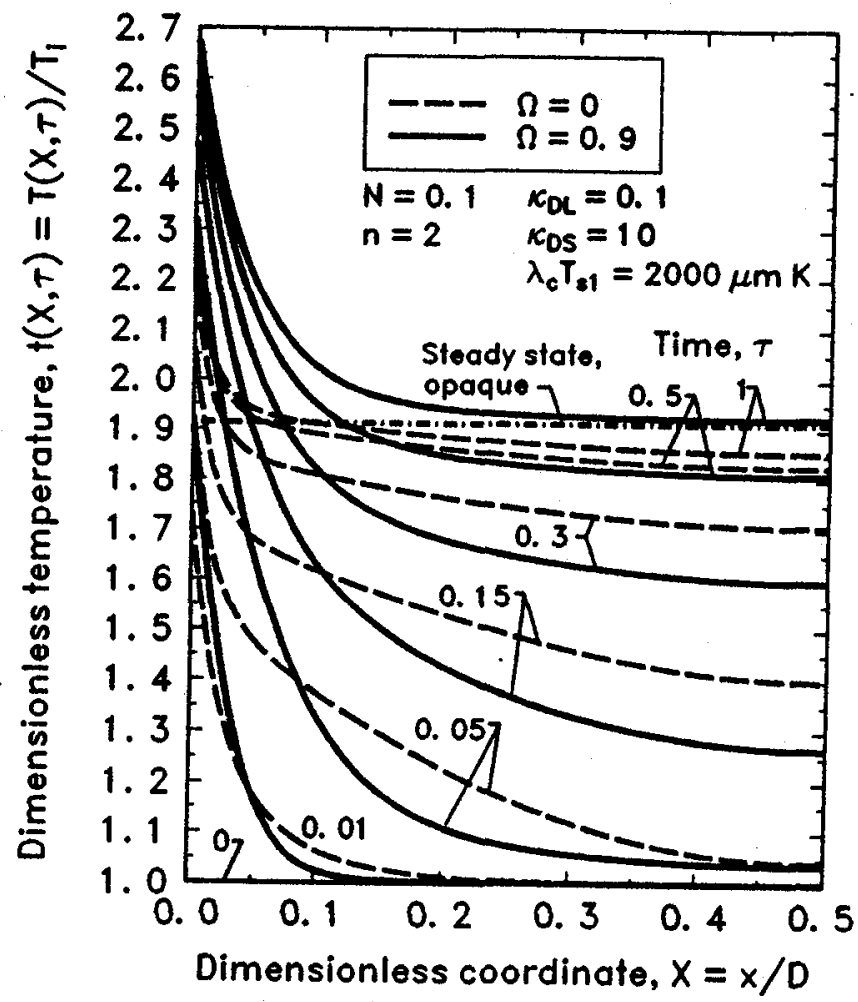

(c) Effect of scattering with $\Omega=0.9$ for two bands with $\mathrm{K}_{\mathrm{DL}}=0.1, \mathrm{~K}_{\mathrm{DS}}=10$, and $\lambda_{\mathrm{c}} \mathrm{T}_{31}=2000$ $\mu \mathrm{m} \mathrm{K}$.

Figure 6. Two-flux transient temperatures in a layer initially at uniform temperature after exposure to convection by a hot gas on both sides, and cold surroundings that produce internal radiative cooling. Parameters: $\mathrm{N}=0.1, \mathrm{n}=2, \tilde{\mathrm{q}}_{\mathrm{r} 1}=\tilde{\mathrm{q}}_{\mathrm{r} 2}=1, \mathrm{H}_{1}=\mathrm{H}_{2}=5, \mathrm{t}_{\mathrm{g} 1}=\mathrm{t}_{\mathrm{g} 2}=4$. 
optical thicknesses from 1 to 10 and for a refractive index of 2 , including internal reflections from diffuse boundaries. Two-band spectral calculations were carried out to demonstrate how the Green's function solution can be applied in spectral bands. The method can be readily extended to include more bands. Illustrative results also demonstrate the effect of isotropic scattering which is included in the present method without additional complexity. The technique is easy to program, has good numerical stability, and requires only modest computing times. It can be used to predict transient spectral behavior in materials subjected to large transient heating by radiation or convection starting from a specified arbitrary temperature distribution.

\section{ACKNOWLEDGMENT}

Consulting help on the Green's function derivation by James A. Pennline of the Mathematical Analysis Section of the NASA Lewis Research Center is gratefully acknowledged.

\section{REFERENCES}

1. Gardon, R., Calculation of Temperature Distributions in Glass Plates Undergoing Heat Treatment, J. Amer. Ceramic Society, Vol. 41, No. 6, pp 200-209, 1958.

2. Fowle, A.A., Strong, P.F., Comstock, D.F. and Sox, C., Computer Program to Predict Heat Transfer Through Glass, AIAA J., Vol. 7, No. 3, pp 478-483, 1969.

3. Su, M.-H. and Sutton, W.H., Transient Conductive and Radiative Heat Transfer in a Silica Window, J. Thermophys. Heat Transfer, Vol. 9, No. 2, pp 370-373, 1995.

4. Field, R.E. and Viskanta, R., Measurement and Prediction of Dynamic Temperatures in Unsymmetrically Cooled Glass Windows, J. Thermophys. Heat Transfer, Vol. 7, No. 4, pp 616623, 1993.

5. Ping, T.H. and Lallemand, M., Transient Radiative-Conductive Heat Transfer in Flat Glasses Submitted to Temperature, Flux and Mixed Boundary Conditions, Int. J. Heat Mass Transfer, Vol. 32, No. 5, pp 795-810, 1989.

6. Siegel, R, Transient Radiative Cooling of a Droplet-Filled Layer, J. Heat Transfer, Vol. 109, No. 1, pp 159-164, 1987.

7. Siegel, R., Transient Heat Transfer in a Semitransparent Radiating Layer with Boundary Convection and Surface Reflections, To be published in Int. J. Heat Mass Transfer.

8. Siegel, R, Two-Flux Method for Transient Radiative Transfer in a Semitransparent Layer," To be published in Int. J. Heat Mass Transfer.

9. Lii, C.C. and Özişik, M.N., "Transient Radiation and Conduction in an Absorbing, Emitting, Scattering Slab with Reflective Boundaries, Int. J. Heat Mass Transfer, Vol. 15, No. 5, pp 1175-1179, 1972.

10. Frankel, J.I., Cumulative Variable Formulation for Transient Conductive and Radiative Transport in Participating Media, J. Thermophys. Heat Transfer, Vol. 9, No. 2, pp 210-218, 1995.

11. Wu, C.-Y. and Ou, N.-R., Transient Two-Dimensional Radiative and Conductive Heat Transfer in a Scattering Medium, Int. J. Heat Mass Transfer, Vol. 37, No. 17, pp 2675-2686, 1994. 
12. Yuen, W.W., Khatami, M. and Cunnington, G.R. Jr., Transient Radiative Heating of an Absorbing, Emitting, and Scattering Material, J. Thermophys. Heat Transfer, Vol. 4, No. 2, pp 193-198, 1990.

13. Tsai, J.-H. and Lin, J.-D., Transient Combined Conduction and Radiation with Anisotropic Scattering, J. Thermophys. Heat Transfer, Vol. 4, No. 1, pp 92-97, 1990.

14. Tsai, C.-F. and Nixon, G., Transient Temperature Distribution of a Multilayer Composite Wall with Effects of Internal Thermal Radiation and Conduction, Numer. Heat Transfer, Vol. 10, pp 95-101, 1986.

15. Siegel, R. and Molls, F.B., Finite Difference Solution for Transient Radiative Cooling of a Conducting Semitransparent Square Region, Int. J. Heat Mass Transfer, Vol. 35, No. 10, pp 2579-2592, 1992.

16. Siddall, R.G., Flux Methods for the Analysis of Radiant Heat Transfer, Proc. of the Fourth Symposium on Flames and Industry, The Institute of Fuel, Paper 16, pp 169-177, 1972.

17. Siegel, R. and Spuckler, C.M., Approximate Solution Methods for Spectral Radiative Transfer in High Refractive Index Layers, Int. J. Heat Mass Transfer, Vol. 37, Suppl. 1, 403-413, 1994.

18. Spuckler, C.M and Siegel, R., Refractive Index Effects on Radiative Behavior of a Heated Absorbing-Emitting Layer, J. Thermophys. Heat Transfer, Vol. 6, No. 4, pp 596-604, 1992.

19. Hildebrand, F.B., Methods of Applied Mathematics, Prentice-Hall, New York, pp 388-389, 1952. 